\title{
Numerical Solution of MHD Incompressible Convection Flow in Channels
}

\author{
Merve Gürbüz ${ }^{1, *}$ and Münevver Tezer-Sezgin ${ }^{2}$ \\ ${ }^{1}$ Department of Management, Baskent University, Ankara, Turkey \\ ${ }^{2}$ Department of Mathematics, Middle East Technical University, Ankara, Turkey \\ E-mail: mervegurbuz@baskent.edu.tr \\ ${ }^{*}$ Corresponding Author
}

Received 03 February 2019; Accepted 03 December 2019;

Publication 18 December 2019

\begin{abstract}
The purpose of this paper is to study numerically the influence of the magnetic field, buoyancy force and viscous dissipation on the convective flow and temperature of the fluid in a square cavity, lid-driven cavity, and lid-driven cavity with an obstacle at the center. The continuity, momentum and energy equations are coupled including buoyancy and magnetic forces, and energy equation contains Joule heating and viscous dissipation. The equations are solved in terms of stream function, vorticity and temperature by using polynomial radial basis function (RBF) approximation for the inhomogeneity and particular solution. The numerical solutions are obtained for several values of Grashof number $(G r)$, Hartmann number $(M)$ for fixed Prandtl number $\operatorname{Pr}=0.71$ and fixed Reynolds number $R e=100$ with or without viscous dissipation. It is observed that in the absence of obstacle, viscous dissipation changes the symmetry of the isotherms, and the dominance of buoyancy force increases with an increase in $G r$, whereas decreases when the intensity of magnetic field increases. The obstacle in the lid-driven cavity causes a secondary flow on its left part. The effect of moving lid is weakened on the flow and isotherms especially for large $G r$ when the cavity contains obstacle.
\end{abstract}

Keywords: MHD flow, RBF, heat transfer, viscous dissipation, obstacle.

European Journal of Computational Mechanics, Vol. 28_5, 411-432.

doi: 10.13052/ejcm2642-2085.2852

(c) 2020 River Publishers 


\section{M. Gürbüz and M. Tezer-Sezgin}

\section{Introduction}

The analysis of the fluid flow and heat transfer in enclosures is one of the most widely studied problems in thermo-fluids area. It has recently attracted the interest of many researchers because of the wide range of industrial applications such as crystal growth, cooling system, air conditioning and solar technology. On the other hand, magnetohydrodynamics (MHD), which focuses on the fluid flows under the magnetic field, has many engineering applications such as MHD pumps, geothermal energy extraction and nuclear fusion. Colaço, Dulikravich \& Orlande (2009) studied the effect of magnetic field on convection heat transfer in a square cavity. They solved the problem in terms of stream function and temperature neglecting viscous dissipation by using RBF approximation. Bakhshan \& Ashoori (2012) analyzed the fluid behavior in a rectangular enclosure under the influence of the slope orientation of magnetic field. They observed that Nusselt number increases with increasing Grashof and Prandtl numbers and decreasing the slope of orientation of magnetic field. MHD free convection flow in a rectangular enclosure problem is solved by modified ADI and SLOR (Successive Line Over Relaxation) methods in (Rudraiah \& Barron, 1995). They indicated that the convective heat transfer rate is reduced when the intensity of magnetic field increases. Aydin (1999) numerically investigated the mixed convection flow in a shear- and buoyancy-driven cavity. The magnetic effect on mixed convection flow in a lid-driven cavity has been added by Kefayati, GorjiBandpy, Sajjadi \& Ganji (2012). The numerical results are obtained by using the Lattice Boltzmann method (LBM). They observed that the increase in the Richardson number increases the heat transfer. Nasrin \& Parvin (2011) have also analyzed MHD mixed convection flow and heat transfer in a lid-driven cavity with sinusoidal wavy bottom surface.

In the above studies, viscous dissipation term has been neglected in the energy equation. Viscous dissipation represents a source of heat because of the friction between the fluid particles. It is characterized by Eckert number. The effect of viscous dissipation was first studied by Gebhart (1962). Later, Kumar (2009) analyzed the heat transfer of the boundary layer flow over stretching plate with viscous dissipation effect in the presence of magnetic field. Kishore, Rajesh \& Verma (2010); Nyabuto, Sigey, Okelo \& Okwoyo (2013) also investigated the effects of viscous dissipation on MHD natural convection flow. They showed that temperature increases with increasing values of Eckert number. Gürbüz \& Tezer-Sezgin (2018) studied the effect on viscous dissipation on MHD flow and heat transfer applying Stokes 
approximation $(R e<<1)$ with small value of Grashof number 90. They found that both Stokes flow and isolines concentrate through the cold wall due to the viscous dissipation effect.

In this study, 2D steady, laminar MHD convection flow of an incompressible and electrically conducting, dissipative viscous fluid in square cavity, lid-driven cavity and lid-driven cavity with a solid obstacle is considered. A uniform magnetic field is applied in the $x$-direction. The governing equations are obtained from Navier-Stokes equations with Lorentz and Buoyancy forces and energy equation including Joule heating and viscous dissipation terms. RBF approximation has been applied to these equations in terms of stream function, vorticity and temperature. The results are validated with the convection flow problem in the absence of magnetic field and viscous dissipation. The main aim of this study is to show the effect of the magnetic field and viscous dissipation on the convective flow in cavities with or without obstacles.

\section{Physical Problem and Mathematical Formulation}

We consider fully developed, steady, laminar flow of an incompressible, electrically conducting fluid in a cross-section of square channel with or without solid obstacle under the influence of a uniform horizontally applied magnetic field. The governing equations are the continuity equation, the momentum equations including buoyancy and Lorentz forces, and the energy equation including Joule heating and viscous dissipation terms (Müller \& Bühler, 2001; Rogers, 1992). The two-dimensional MHD convection flow is represented with Poisson's type equations in terms of stream function, temperature and vorticity

$$
\begin{aligned}
\nabla^{2} \psi= & -\omega \\
\nabla^{2} \omega= & \operatorname{Re}\left(u \frac{\partial \omega}{\partial x}+v \frac{\partial \omega}{\partial y}\right)+M^{2} \frac{\partial v}{\partial x}-\frac{G r}{\operatorname{Re}} \frac{\partial T}{\partial x} \\
\nabla^{2} T= & \operatorname{Pr} \operatorname{Re}\left(u \frac{\partial T}{\partial x}+v \frac{\partial T}{\partial y}\right)-M^{2} E c P r v^{2} \\
& -\operatorname{EcPr}\left(2\left[\left(\frac{\partial u}{\partial x}\right)^{2}+\left(\frac{\partial v}{\partial y}\right)^{2}\right]+\left(\frac{\partial v}{\partial x}+\frac{\partial u}{\partial y}\right)^{2}\right)
\end{aligned}
$$

where $u=\frac{\partial \psi}{\partial y}$ and $v=-\frac{\partial \psi}{\partial x}$. The non-dimensional parameters $R e$, $M$ and $\operatorname{Pr}$ are Reynolds number, Hartmann number and Prandtl number, 
respectively, defined as $R e=L U_{0} / \nu, M=L \mu H_{0} \sqrt{\sigma / \rho \nu}$, and $\operatorname{Pr}=$ $\rho c_{p} \nu / \lambda$. Grashof number $G r=g \beta\left(T_{\text {hot }}-T_{\text {cold }}\right) L^{3} / \nu^{2}$ is the ratio of buoyancy force to viscous force. Eckert number $E c=U_{0}^{2} /\left(c_{p}\left(T_{\text {hot }}-T_{\text {cold }}\right)\right)$ can be interpreted as the ratio of kinetic energy to the accumulated enthalpy. Here, $\nu, \sigma, \mu, \rho, c_{p}, \beta, \lambda$ and $\mathbf{g}$ are kinematic viscosity, electrical conductivity, magnetic permeability, the density, specific heat, thermal expansion coefficient, the thermal conductivity of the fluid and the gravitational acceleration vector, respectively.

\section{Radial Basis Function (RBF) Approximation}

All the Equations (1-3) are Poisson's type equations considering the right hand sides $f(x, y)$ as inhomogeneity. We solve them iteratively by using RBF approximation. In this method (Chen, Fan \& Wen, 2012), the inhomogeneity $f(x, y)$ and the particular solution $\hat{u}$ of a partial differential equation $L u(x, y)=f(x, y)$ are approximated

$$
f(x, y) \simeq \sum_{j=1}^{n} a_{j} \varphi_{j}(r) \quad \text { and } \quad \hat{u}(x, y)=\sum_{j=1}^{n} a_{j} \Psi_{j}(r), \quad(x, y) \in \Omega
$$

where $r=\sqrt{\left(x-x_{j}\right)^{2}+\left(y-y_{j}\right)^{2}}$ is the Euclidean distance and $n$ is the number of unknown coefficients, $\varphi_{j}(r)$ 's are the radial basis functions. Boundary condition $B u(x, y)=g(x, y)$ is going to be satisfied by a particular solution $\hat{u}$ which in turn is also approximated by RBFs as $\sum_{j=1}^{n} a_{j} B \Psi_{j}(r)=g(x, y),(x, y) \in \partial \Omega$ where $\Psi_{j}$ 's are linked to $\varphi_{j}$ 's through $L \Psi_{j}(r)=\varphi_{j}(r)$.

The undetermined coefficients $a_{j}$ in the approximation (4) are found collocating the unknown functions $f(x, y)$ and $g(x, y)$ at $N+L=n$ collocation points $\left(x_{i}, y_{i}\right)$ on the boundary and interior of the domain as

$$
\begin{aligned}
& \sum_{j=1}^{n} a_{j} B \Psi_{j}\left(r_{k}\right)=g\left(x_{k}, y_{k}\right), \quad 1 \leq k \leq N, \\
& \sum_{j=1}^{n} a_{j} \varphi_{j}\left(r_{l}\right)=f\left(x_{l}, y_{l}\right), \quad 1+N \leq l \leq n
\end{aligned}
$$

which are combined to give one linear system $[A]\{a\}=\{b\}$ for the solution vector $\{a\}=\left[\begin{array}{lll}a_{1} & \cdots & a_{n}\end{array}\right]^{T}$. The coefficient matrix and the right hand side 
vector are given as

$[A]=\left[\begin{array}{cccc}B \Psi_{1}\left(r_{1}\right) & B \Psi_{2}\left(r_{1}\right) & \cdots & B \Psi_{n}\left(r_{1}\right) \\ \vdots & \vdots & \ddots & \vdots \\ B \Psi_{1}\left(r_{N}\right) & B \Psi_{2}\left(r_{N}\right) & \cdots & B \Psi_{n}\left(r_{N}\right) \\ \varphi_{1}\left(r_{N+1}\right) & \varphi_{2}\left(r_{N+1}\right) & \cdots & \varphi_{n}\left(r_{N+1}\right) \\ \vdots & \vdots & \ddots & \vdots \\ \varphi_{1}\left(r_{n}\right) & \varphi_{2}\left(r_{n}\right) & \cdots & \varphi_{n}\left(r_{n}\right)\end{array}\right]_{n \times n} \quad,\{b\}=\left[\begin{array}{c}g\left(x_{1}, y_{1}\right) \\ \vdots \\ g\left(x_{N}, y_{N}\right) \\ f\left(x_{N+1}, y_{N+1}\right) \\ \vdots \\ f\left(x_{n}, y_{n}\right)\end{array}\right]_{n \times 1}$

Solution of this whole system provides the coefficients $a_{j}$ 's, $1 \leq j \leq n$, and the approximate solution $\hat{u}(x, y)=\sum_{j=1}^{n} a_{j} \Psi_{j}(r)$. Now, application to MHD convection Equations (1-3) gives $\hat{u}$ representing $\psi, \omega$ or $T$, respectively.

\subsection{Application to MHD Convection Flow Equations}

Polynomial type $\operatorname{RBF} \varphi(r)=1+r$ is used for the approximations of the inhomogeneity and boundary function which gives $\Psi(r)=\frac{r^{2}}{4}+\frac{r^{3}}{9}$ from backward integration in $\nabla^{2} \Psi=\varphi$ for approximating the solution itself. The system of Equations (1-3) are solved with an iterative process. Given an initial estimate for vorticity, stream function Equation (1) is solved. The unknown vorticity wall conditions are obtained from the finite difference discretization of stream function equation which uses interior values (Gürbüz \& Tezer-Sezgin, 2015). Then, we solve the vorticity Equation (2). The energy Equation (3) is solved with the use of the new values of velocity components and initial estimate for temperature. The iteration continues until a preassigned tolerance $(\epsilon)$ is reached between two successive iterations. In each iterations, all the space derivatives are computed by using coordinate matrix $\varphi$ which is constructed by $\varphi_{i j}=1+r_{i j}$

$$
\frac{\partial D}{\partial x}=\frac{\partial \varphi}{\partial x} \varphi^{-1} D, \quad \frac{\partial D}{\partial y}=\frac{\partial \varphi}{\partial y} \varphi^{-1} D
$$

where $D$ denotes $u, v, \omega$ and $T$.

\section{Numerical Results and Discussion}

The steady convection flow in a square cavity, a lid-driven cavity and a liddriven cavity with an obstacle at the center under the influence of a uniform 
horizontally applied magnetic field is analyzed by using RBF approximation. Convergence tolerance is tested and $10^{-4}$ accuracy is sufficient enough to show the behavior of the flow and the temperature. Also, we use relaxation parameters $\alpha$ and $\beta, 0 \leq \alpha, \beta \leq 1$ for the vorticity and the temperature, respectively, to accelerate the convergence.

\subsection{Square Cavity}

First, the Equations (1-3) are solved iteratively with initial estimates for $\omega$ and $T$ and the boundary conditions shown in Figure 1.

Numerical results are obtained for several values of $G r\left(10^{3} \leq G r \leq 10^{5}\right)$ and moderate Hartmann number values $M(0 \leq M \leq 50)$ with fixed $\operatorname{Pr}=0.71, R e=100$ to see the effect of viscous dissipation by taking $E c=0$ or $E c=1$. The boundary is discretized by taking $N=80$ points and sufficient number of interior points to demonstrate the flow, temperature and pressure behaviors in the cavity. The numerical results are shown in terms of streamlines, isotherms and equivorticity contours.

In Figure 2, we show convection flow and temperature behavior in the square cavity for increasing values of $G r$ neglecting applied magnetic field $(M=0)$ and viscous dissipation $(E c=0)$ which are in good agreement with the results in (Al-Najem, Khanafer \& El-Refaee, 1998). As $G r$ increases, the stream function value also increases and center vortex tends to be divided into two vortices. Similarly, vorticity is divided at the center of the cavity forming vortices and boundary layers near the left and right walls. Isotherms variation

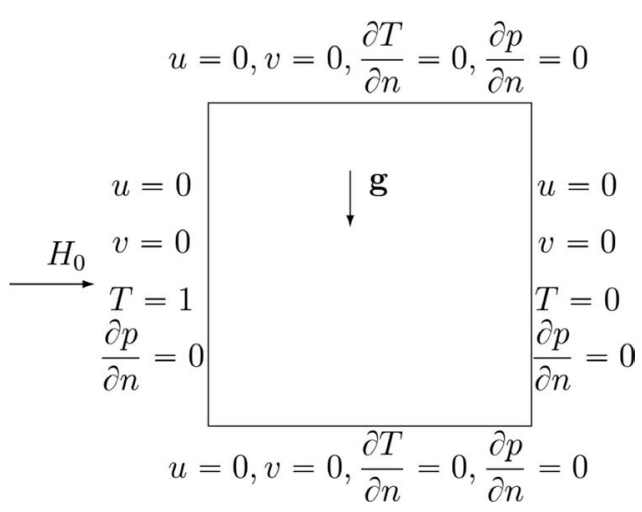

Figure 1 Square cavity with boundary conditions. 


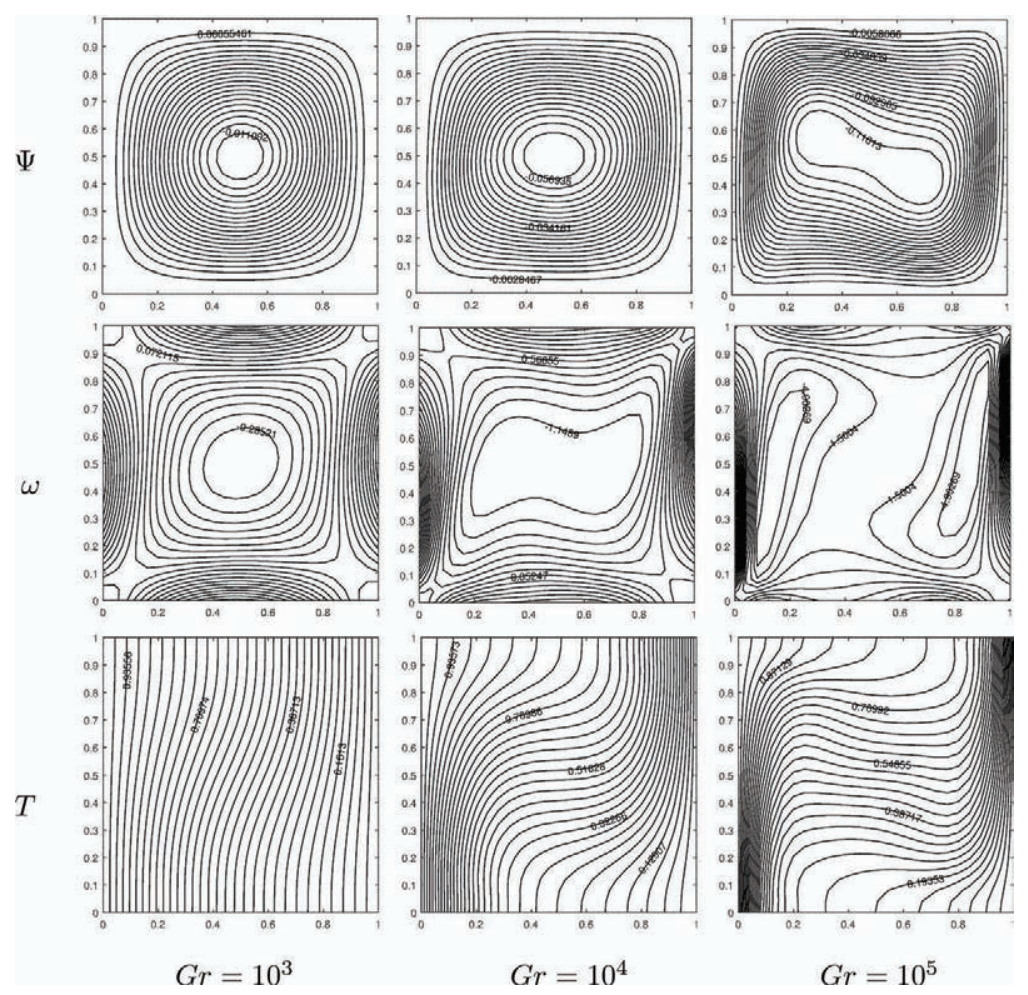

Figure 2 The effect of Grashof number on $\Psi, \omega$ and $T$ at fixed $M=0, E c=0$ (Square cavity).

is more pronounced with an increase in Grashof number due to dominance of buoyancy effect.

Figure 3 indicates the effect of the magnetic field on the flow and temperature profiles for fixed $R e=100, G r=10^{4}$ and $E c=0$. Results are in well agreement with the ones obtained in (Al-Najem et al., 1998; Colaço, Dulikravich \& Orlande, 2009). Hartmann number effect on the flow is the boundary layer formation near the walls which are perpendicular to the applied magnetic field (Hartmann layers). External magnetic field impact is most pronounced on isotherms. It reduces the dominance of convective heat transfer from the left wall to the right wall (which was observed for large $G r$ number). When $M$ reaches to the value of 50 , the temperature comes back to the uniform distribution between the vertical walls which indicates that heat conduction becomes dominant. 

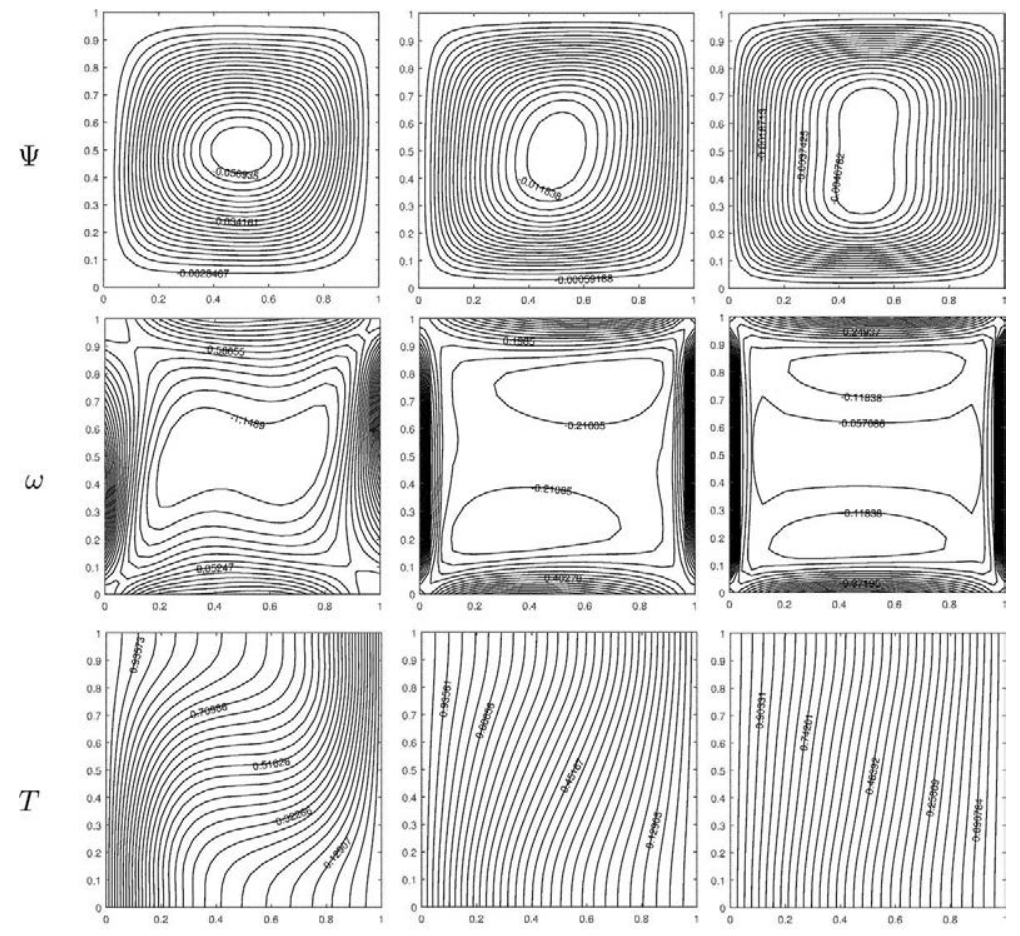

$$
M=0
$$

$M=30$

$$
M=50
$$

Figure 3 The effect of Hartmann number on $\Psi, \omega$ and $T$ at fixed $G r=10^{4}, E c=0$ (Square cavity).

When we add viscous dissipation term in the energy equation by taking $E c=1$, flow characteristics are shown in Figures 4-5 with respect to $G r$ increase and $M$ increase. Main vortex of the streamlines moves to the right bottom corner forming boundary layer with an increase in $G r$ up to $10^{5}$. As Grashof number is taken up to $10^{5}$, the symmetry of vorticity and isotherms are altered, and they form boundary layers near the cold wall. Temperature values increase through the center of the cavity, but decrease through the right cold wall showing heat transfer from the left wall through upper adiabatic wall and sharp decrease to the cold wall. This means that the thermal convection is completely changed for dissipative viscous fluid only when $G r$ is reached to $10^{5}$ which can be seen from the third row of Figure 4 with the third row of Figure 2.

In Figure 5, the impacts of both the magnetic field and the buoyancy force are searched on the solution for dissipative viscous fluid. For $G r=10^{4}$ the 

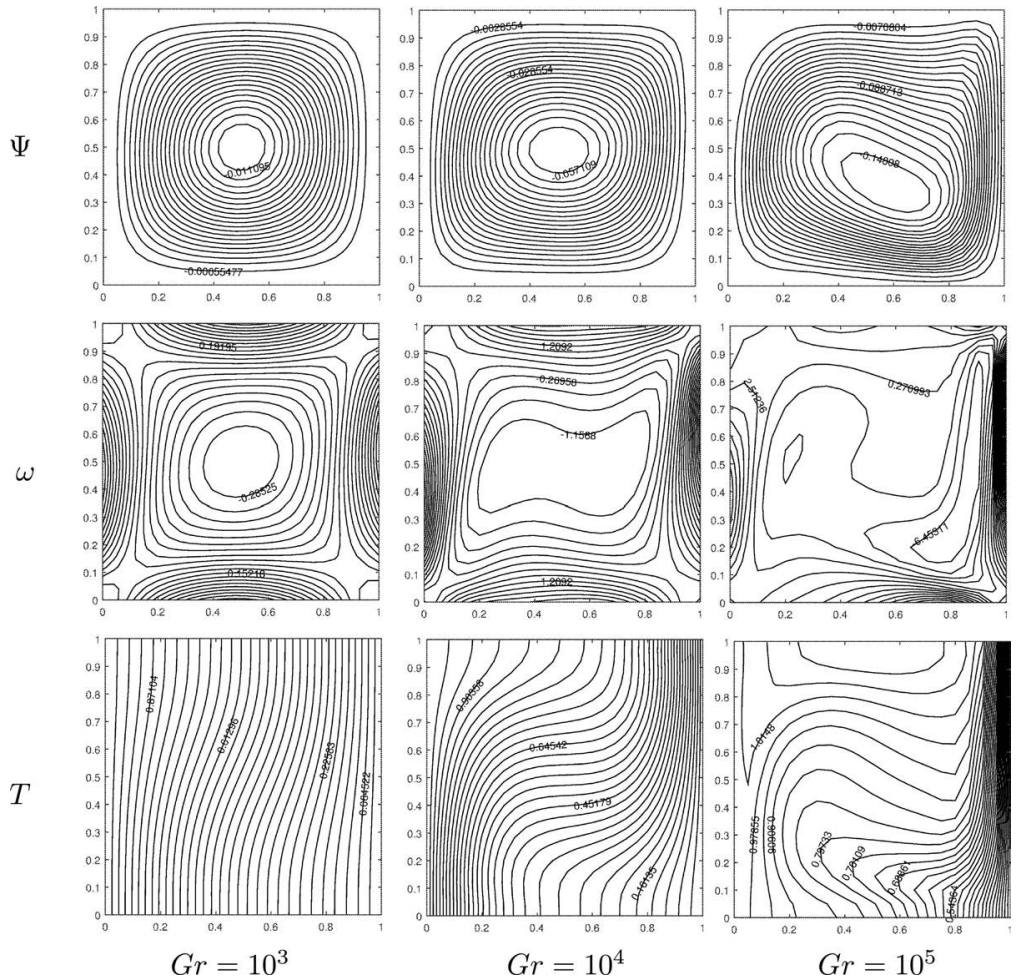

Figure 4 The effect of Grashof number on $\Psi, \omega$ and $T$ at fixed $M=0, E c=1$ (Square cavity).

magnetic field effect on the dissipative flow is the same as in the case of $E c=0$ (Figure 3). When $G r$ reaches to $10^{5}$ viscous dissipation effect starts to be observed. Streamlines, isotherms and equivorticity lines all concentrate near the right bottom corner and form boundary layer near the cold right wall.

\subsection{Lid-Driven Cavity}

The proposed method has been applied to the convection flow in lid-driven cavity under the effect of a uniform horizontally applied magnetic field. The problem configuration and the boundary values are shown in Figure 6. The numerical results are obtained for Hartmann number values in the range $(0-80)$ and Grashof number is taken up to $10^{4}\left(10^{2} \leq G r \leq 10^{4}\right)$ for fixed Reynolds number value of 100 . Solution is depicted in terms of streamlines, vorticity contours and isotherms. 


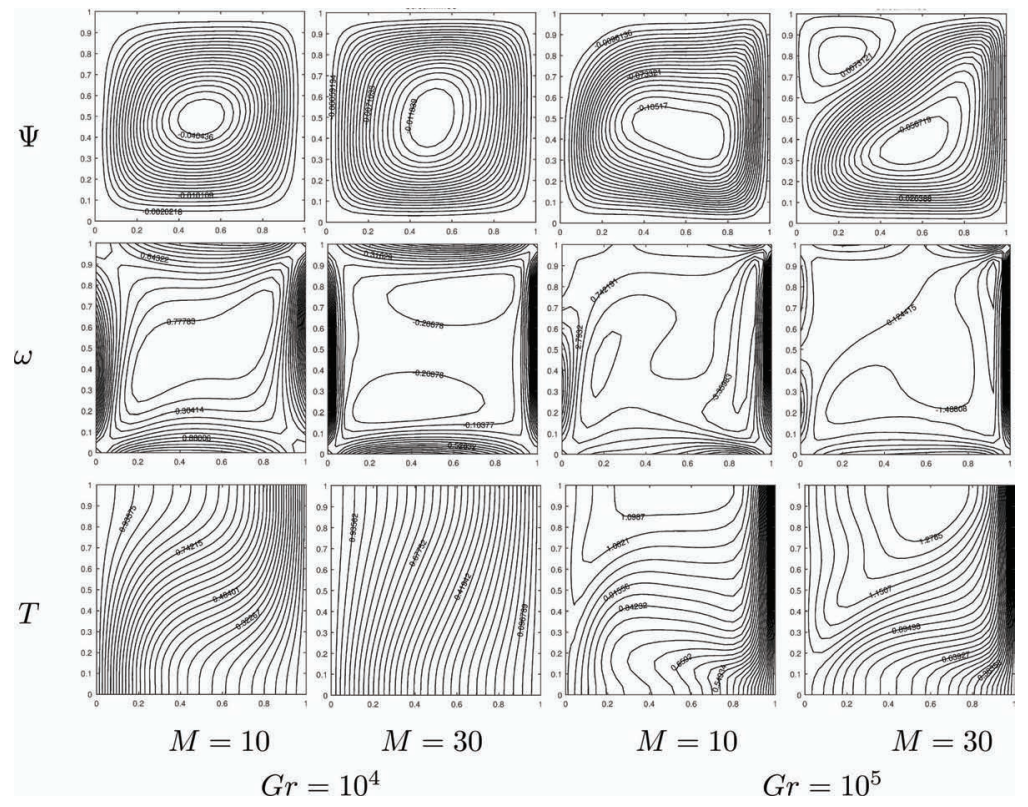

Figure 5 The effects of magnetic field and buoyancy on $\Psi, \omega$ and $T$ at fixed $E c=1$, $G r=10^{4}$ and $G r=10^{5}$ (Square cavity).

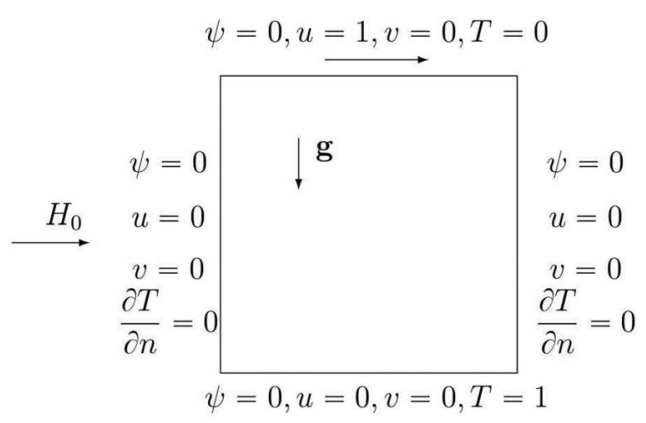

Figure 6 Lid-driven cavity.

In Figure 7, the increase in the Grashof number is considered on the flow and temperature behaviors in the absence of the magnetic field and viscous dissipation effects. These results are in well agreement with the ones obtained in Yapicı \& Obut (2015), since $E c=0$ corresponds to the heat transfer without viscous dissipation. As $G r$ increases, central vortex of stream function moves through the center of the cavity with an increasing magnitude. 

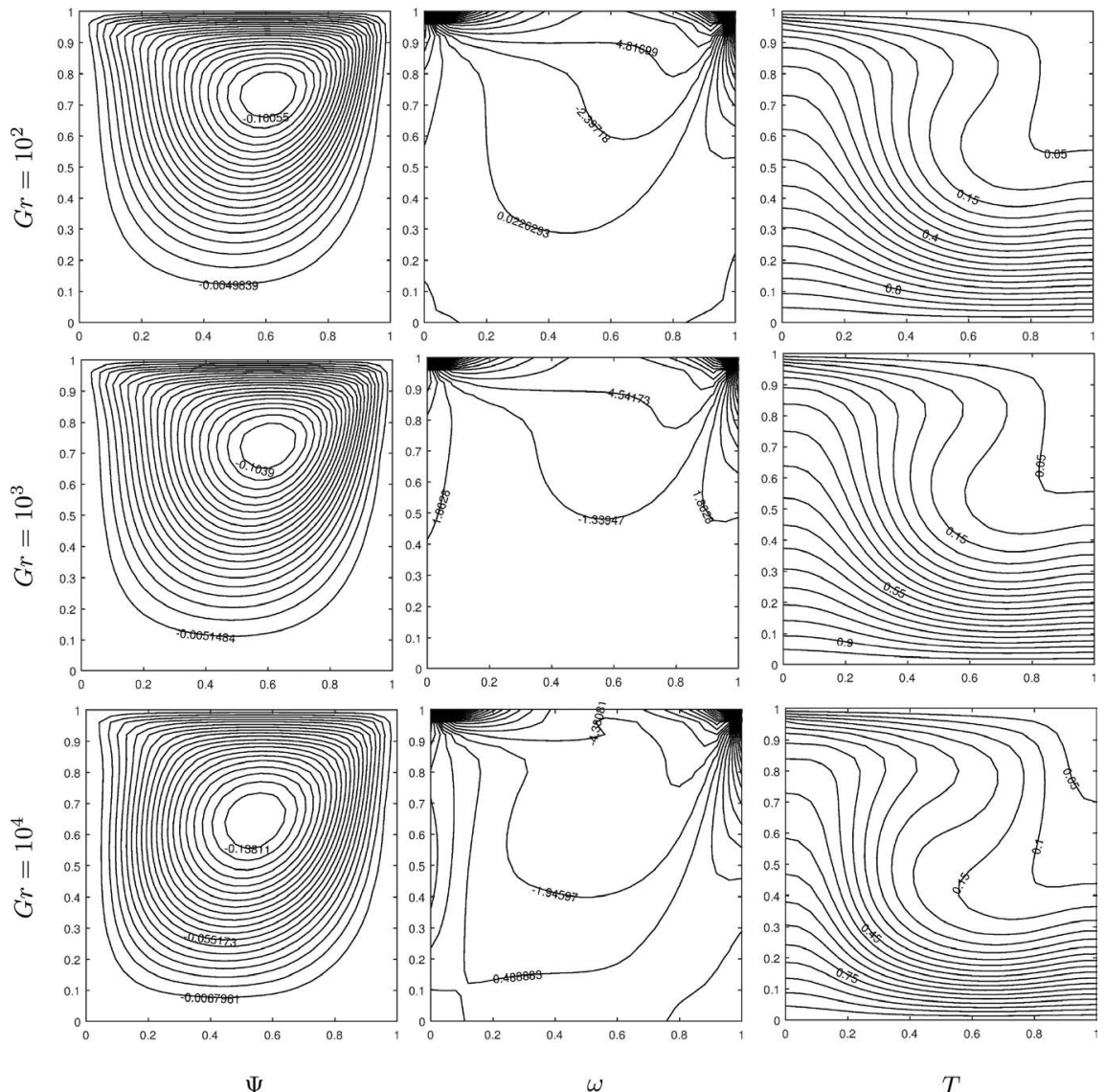

$\omega$

$T$

Figure 7 The effect of Grashof number on the flow and temperature for $M=0, E c=0$, $R e=100$ (lid-driven).

Lid-driven effect can be seen on the flow. Isotherms bend through the center and concentrate near the hot bottom wall since buoyancy force dominates the flow ( $G r$ contains thermal expansion coefficient and thermal conductivity of the fluid).

Figure 8 presents the variation of the Hartmann number for fixed $G r=$ $10^{3}$ and $E c=0$. It is observed that as the magnetic field intensity increases, the main flow shifts through the moving top lid with a decrease in the magnitude. With a further increase in $M$ flow concentrates in front of the moving lid and forming a side layer which is an expected behavior of MHD flow. 

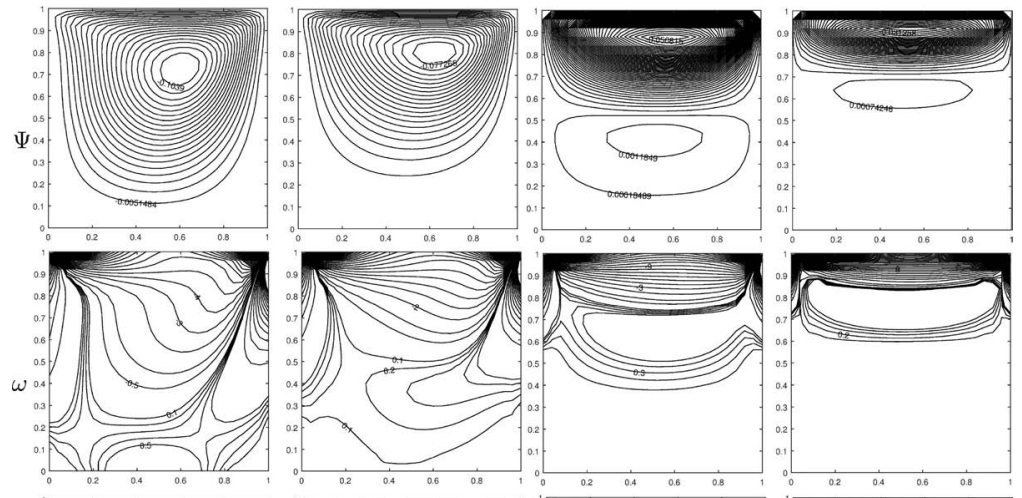

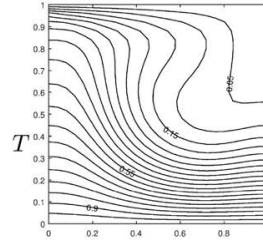

$M=0$

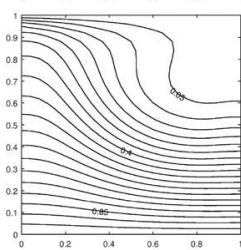

$M=10$

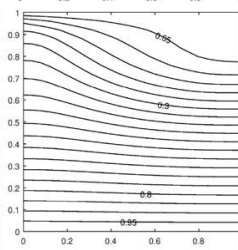

$M=30$

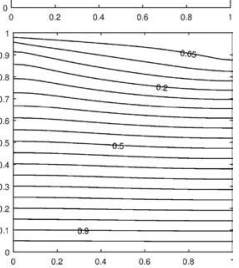

$M=80$

Figure 8 The effect of Hartmann number on the flow and temperature for $G r=10^{3}, E c=$ $0, R e=100$ (lid-driven).

For larger $M$ even secondary flow with small magnitude starts to develop and the rest of the cavity is almost stagnant. Magnetic field effect on isotherms is opposite to the effect of $G r$. This means that further increase in $M$ causes the convection to be suppressed as in the case of a small $G r$.

The effect of viscous dissipation on the MHD convection flow can be seen in Figures $9-10$ by taking $E c=1$. Figure 9 depicts the variations of $G r$ on the flow and temperature when $E c=1$. One can see that boundary layer is developed near the moving top lid when $G r$ is increased, and the increase in $G r$ causes the extension of circulation of the fluid through the center of the cavity as in the case of $E c=0$. Thus, viscous dissipation does not cause a significant change on the flow when we compare to Figure 7. On the other hand, isotherms tend to bend inside the cavity between adiabatic walls with the effects of moving lid, and an increase in $G r$. Viscous dissipation effect together with the buoyancy force cause the temperature values to drop suddenly near the upper lid forming boundary layer instead of concentrating through the hot wall as in the case of $E c=0$ (Figure 7).

The effects of both magnetic field and viscous dissipation can be seen in Figure 10. The flow behavior is the same as in the case $E c=0$ (Figure 8). 

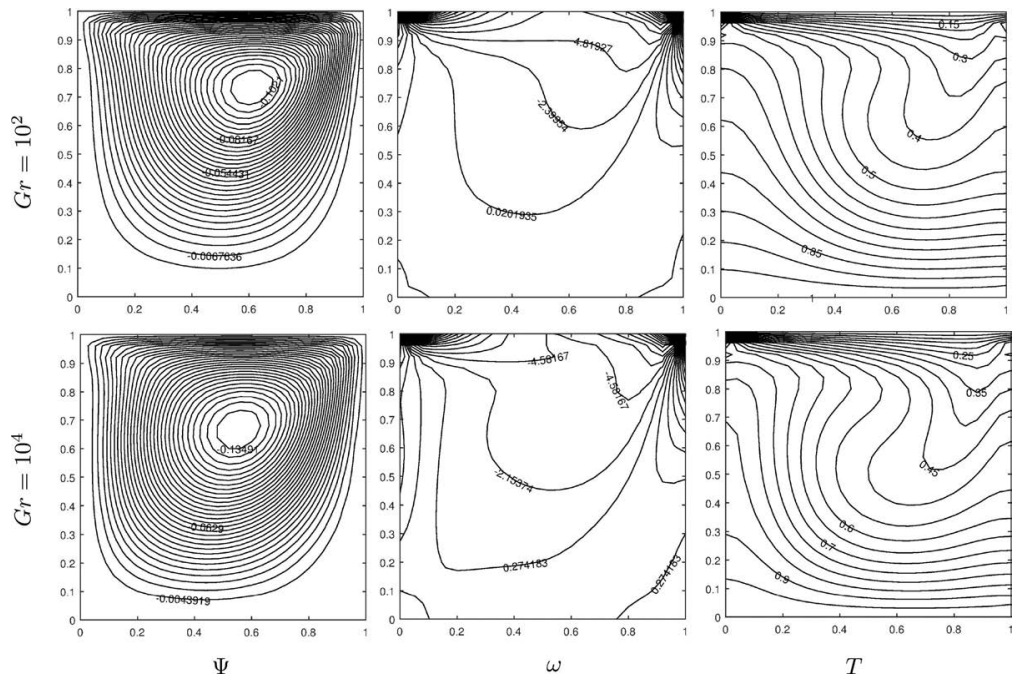

Figure 9 The effect of Grashof number on the flow and temperature for $M=0, E c=1$ (lid-driven).
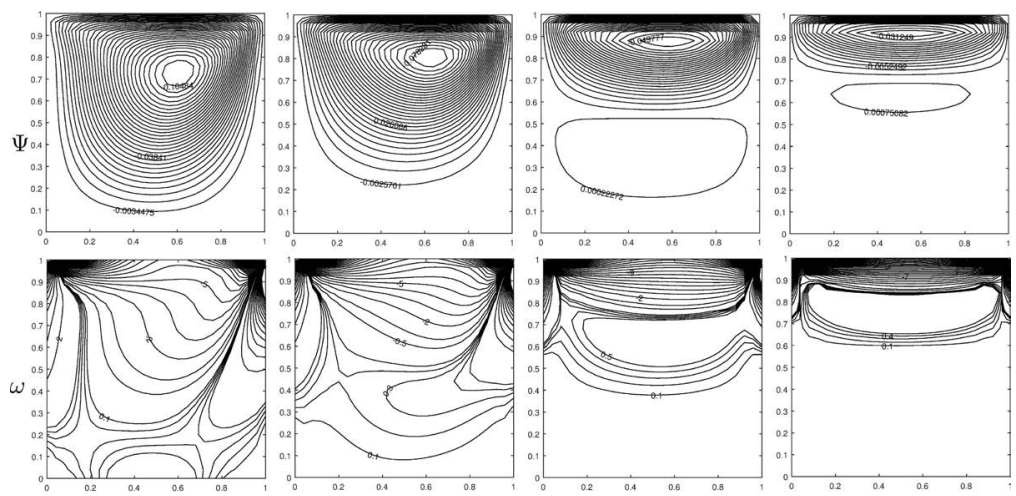

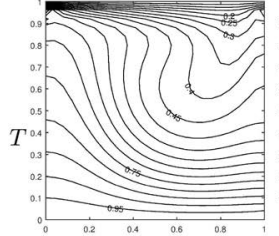

$M=0$

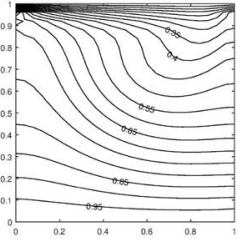

$M=10$

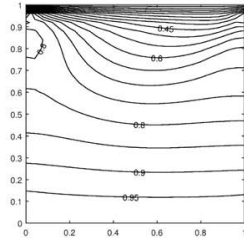

$M=30$

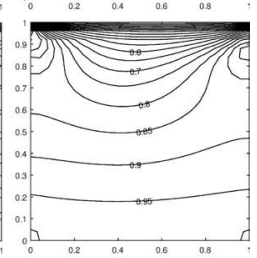

$M=80$

Figure 10 The effect of Hartmann number on the flow and temperature for $G r=10^{3}$, $R e=100, E c=1$ (lid-driven). 


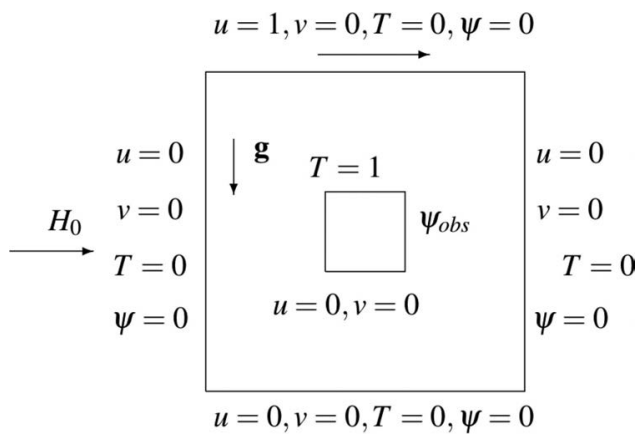

Figure 11 Lid-driven cavity with obstacle.
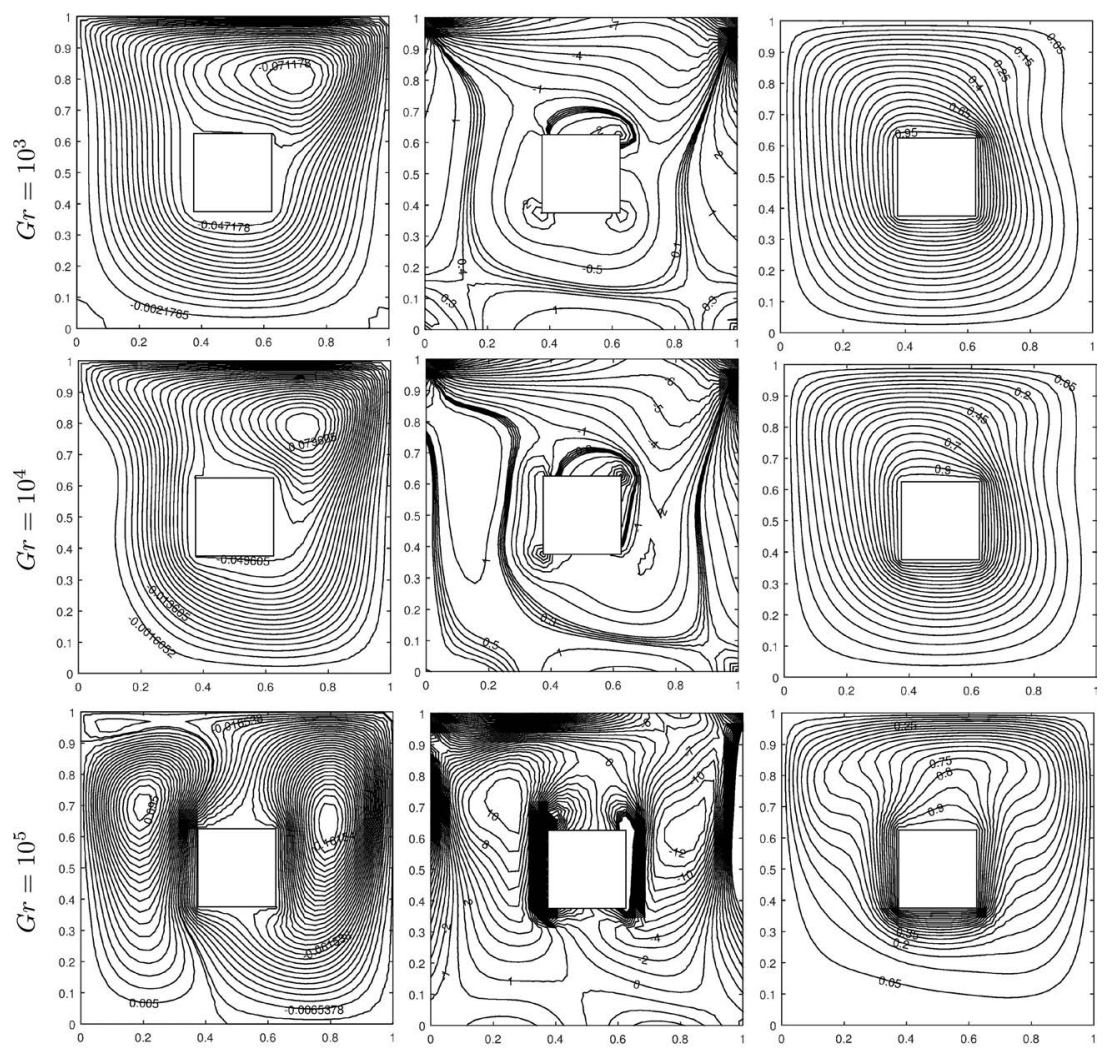

$\Psi$

$\omega$

$T$

Figure 12 The effect of Grashof number on the flow and temperature for $M=0, E c=0$, $R e=100$ (lid-driven with obstacle). 

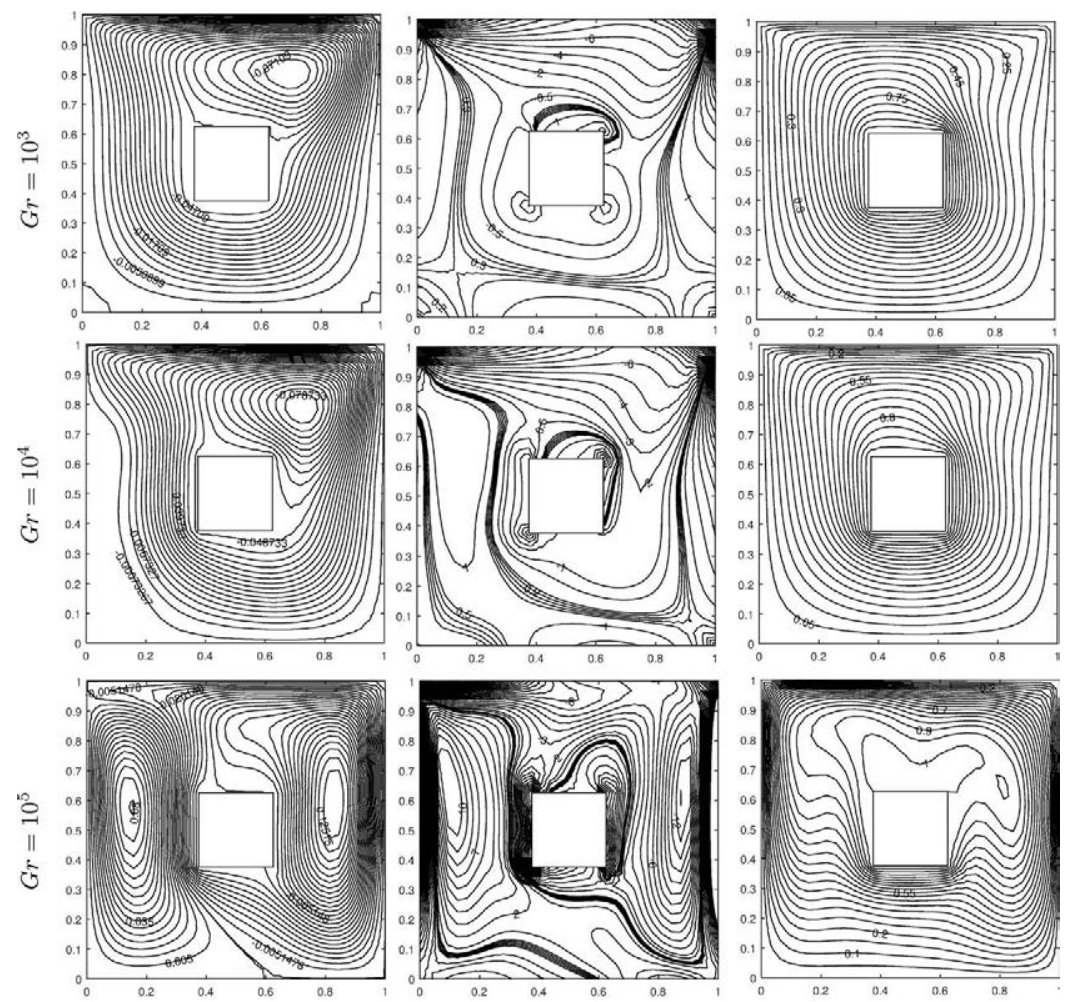

$\omega$

$T$

Figure 13 The effect of Grashof number on the flow and temperature for $M=0, E c=1$, $R e=100$ (lid-driven with obstacle).

However, viscous dissipation impact is most pronounced on the temperature contours. Magnetic field has a cooling effect on isotherms pushing the heat through the moving lid and forming a boundary layer near the cold wall.

\subsection{Lid-Driven Cavity with an Obstacle}

As a last test problem, the MHD convection flow is considered in a liddriven cavity containing a square obstacle with heated walls at the center of the cavity. The outer walls of the cavity are taken as cooled. The external magnetic field still applies horizontally. The geometry of the problem and related boundary conditions are shown in Figure 11. The effect of the blockage on the flow and fluid temperature is studied again for problem parameters 

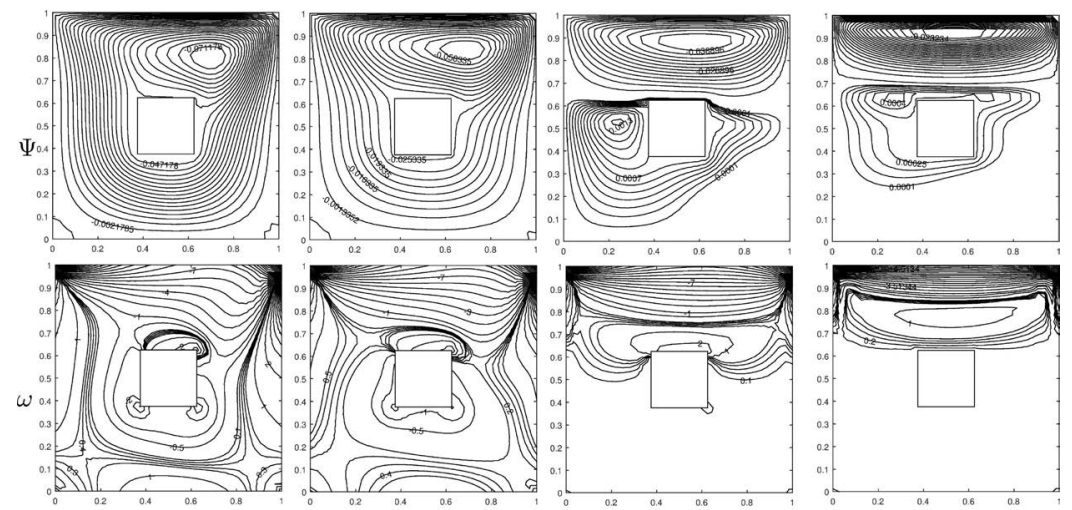

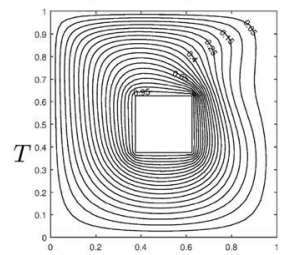

$M=0$

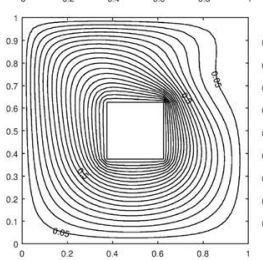

$M=10$

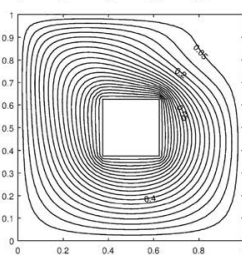

$M=30$

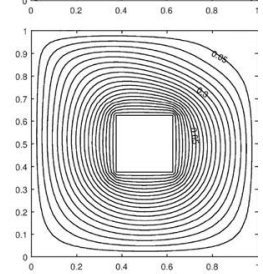

$M=80$

Figure 14 The effect of Hartmann number on the flow and temperature for $G r=10^{3}$, $E c=0, R e=100$ (lid-driven with obstacle).

$0 \leq M \leq 80,10^{3} \leq G r \leq 10^{5}, R e=100$ with $E c=0$ or $E c=1$. The boundary conditions for the stream function on the obstacle $\left(\psi_{\text {obs }}\right)$ are taken by looking at the average $\psi$ values at the center of the cavity in the absence of the obstacle and heat transfer.

As $G r$ increases when $M=0, E c=0$, a secondary flow is developed on the left of the blockage. Fluid flows near the left and right sides of the obstacle in terms of layers and the effect of moving lid is weakened especially when $G r$ is further increased to $G r=10^{5}$. Isotherms concentrate near the hot obstacle walls with heat transfer from the upper obstacle wall through the upper moving cold lid (Figure 12). These results are in well agreement with the results in (Islam, Sharif \& Carlson, 2012).

Figure 13 shows that the effect of $G r$ on the flow and the temperature when the viscous dissipation is present $(E c=1)$. When it is compared with Figure 12, one can see that viscous dissipation does not cause a significant change on the flow as in the case of cavity without an obstacle. However, viscous dissipation effect causes the isotherms to concentrate evenly through 

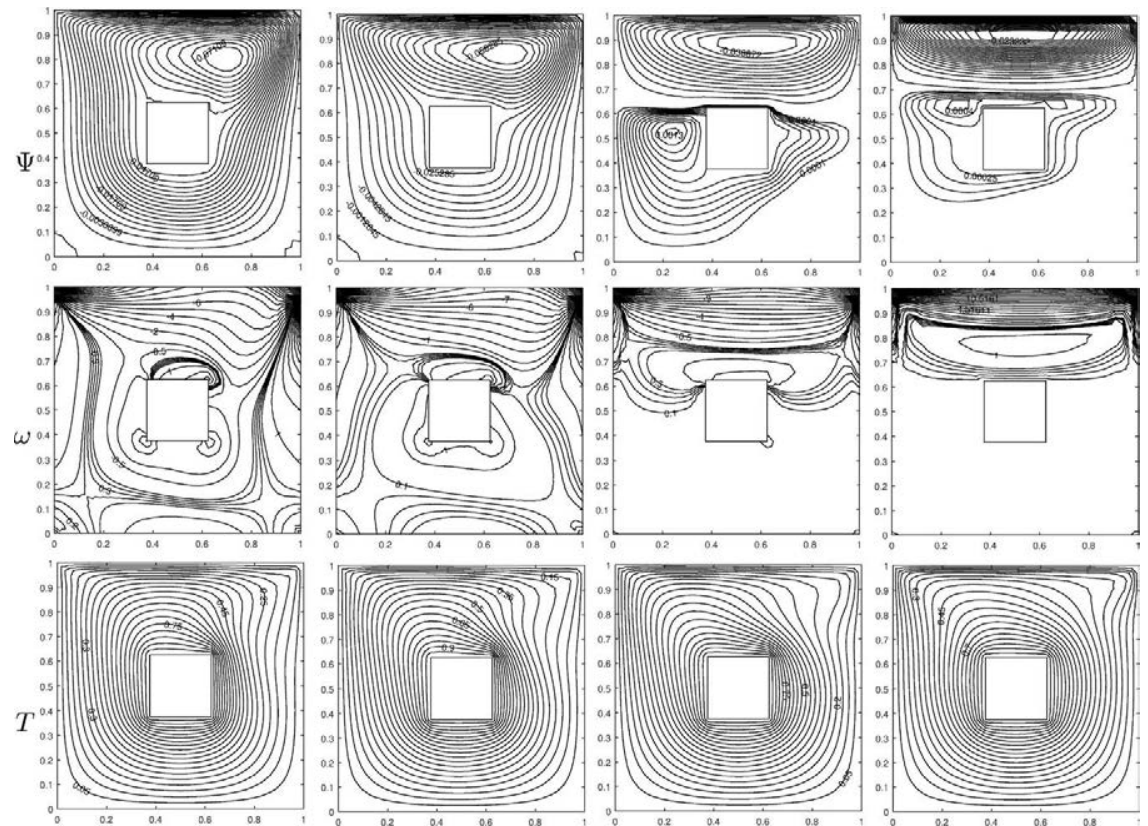

$M=0$

$M=10$

$M=30$

$M=80$

Figure 15 The effect of Hartmann number on the flow and temperature for $G r=10^{3}$, $E c=1, R e=100$ (lid-driven with obstacle).
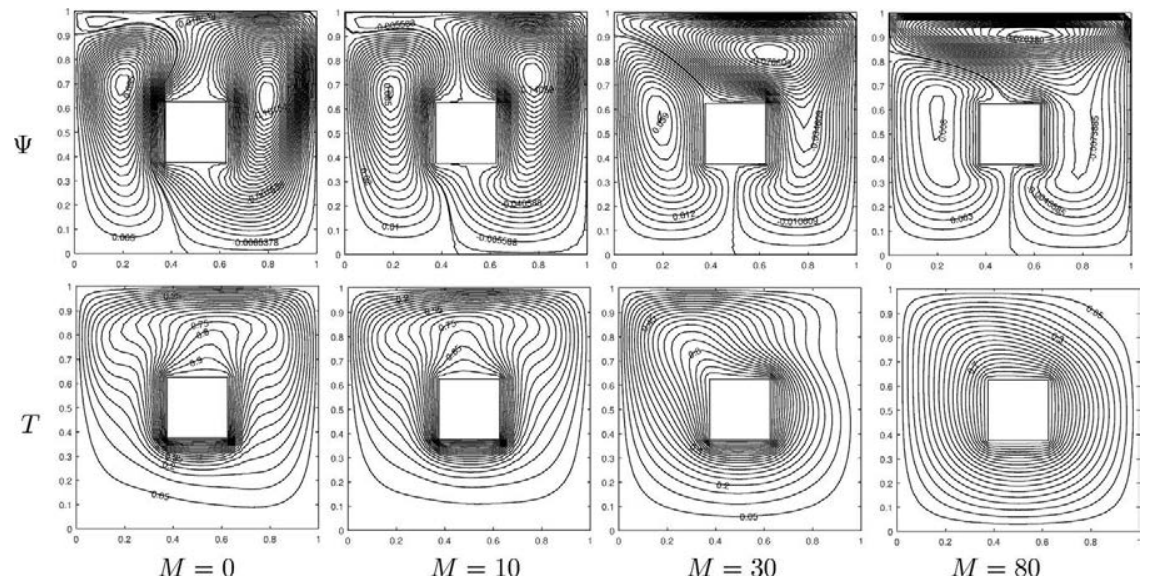

Figure 16 The effect of Hartmann number on the flow and temperature for $G r=10^{5}$, $E c=0, R e=100$ (lid-driven with obstacle). 

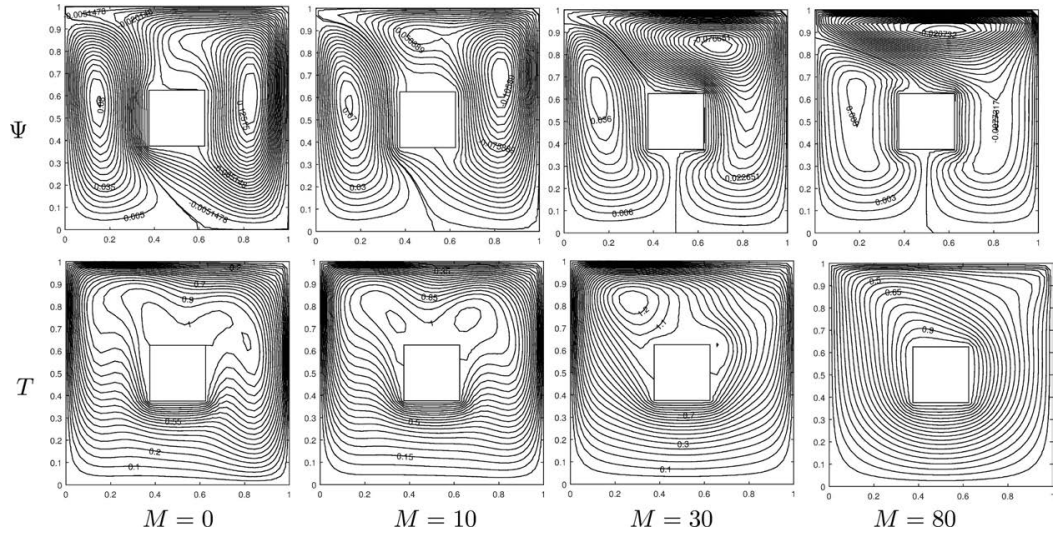

Figure 17 The effect of Hartmann number on the flow and temperature for $G r=10^{5}$, $E c=1, R e=100$ (lid-driven with obstacle).

the upper moving cold lid and the side walls of the cavity instead of concentrating around the hot walls of the obstacle as in the case $E c=0$ shown in Figure 12.

The effect of the increase in the Hartmann number is similar to the effect in the absence of obstacle when $G r=10^{3}$ is fixed and $E c=0$. That is main flow is shifted through the upper lid and when $M$ is further increased, a secondary flow around the obstacle is developed with a vortex on the left upper corner of the obstacle. The magnitude of the flow is decreased when $M$ is increased. Both the flow and the temperature are out of the effect of moving upper lid when external magnetic field influence is further increased as can be seen in Figure 14.

For a fixed moderate $G r=10^{3}$, the effect of increasing $M$ is the same with $E c=0$ or $E c=1$ when the Figures 14-15 are compared. But, for larger $G r=10^{5}$, the increase in the Hartmann number $M$ is more pronounced on isotherms for $E c=1$ as their concentration on the obstacle walls leave their place to a uniform heat transfer from inner to outer cavity walls (Figures 16-17).

\section{Conclusion}

The MHD convection flow in a square cavity and a lid-driven cavity are solved by using polynomial RBF approximation. This numerical technique is easy to implement compared to other domain discretization methods since 
the points can be arranged arbitrarily. Also, the solution is obtained in a considerably low computational cost. We consider the effects of viscous dissipation, magnetic field and buoyancy force on the convection flow. As $G r$ increases for cavities without obstacle, isotherms undergo an inversion in the cavity due to the convection dominance. However, heat conduction becomes dominant with an increasing intensity of the magnetic field. When the viscous dissipation is present, flow behavior does not change significantly, but temperature variation is more pronounced. Isotherms values drop suddenly near the upper lid forming boundary layer in a lid-driven cavity. For square cavity problem viscous dissipation effect is suddenly observed when $\mathrm{Gr}$ reaches to $10^{5}$. Symmetric behavior of the flow and temperature are destroyed. When the lid-driven cavity includes an obstacle at the center, the effect of the moving lid is weakened for large Grashof number and as Hartmann number is increased, viscous dissipation causes a uniform heat transfer from hot obstacle walls to cold cavity walls again for large $G r$ values.

\section{References}

Al-Najem, N. H., Khanafer, K. M. \& El-Refaee, M. M. (1998). Numerical study of laminar natural convection in tilted enclosure with transverse magnetic field. International Journal of Numerical Methods for Heat \& Fluid Flow, 8, 651-672.

Aydin, O. (1999). Aiding and opposing mechanism of mixed convection in a shear- and buoyancy-driven cavity. International Communication in Heat and Mass Transfer, 26, 1019-1028.

Bakhshan, Y. \& Ashoori, H. (2012). Analysis of a fluid behavior in a rectangular enclosure under the effect of magnetic field. World Academy of Science, Engineering and Technology, 61, 637-641.

Chen, C. S., Fan, C. M. \& Wen, P. H. (2012). The method of approximate particular solutions for solving certain partial differential equations. Numerical Methods for Partial Differential Equations, 28, 506-522.

Colaço, M. J., Dulikravich, G. S. \& Orlande, H. R. B. (2009). Magnetohydrodynamic simulations using radial basis functions. International Journal of Heat and Mass Transfer, 52, 5932-5939.

Gebhart, B. (1962). Effects of viscous dissipation in natural convection. Journal of Fluid Mechanics, 14, 225-232.

Gürbüz, M. \& Tezer-Sezgin, M. (2015). MHD Stokes flow in lid-driven cavity and backward-facing step channel, European Journal of Computational Mechanics, 24(6), 279-301. 
Gürbüz, M. \& Tezer-Sezgin, M. (2018). MHD Stokes flow and heat transfer in a lid-driven square cavity under horizontal magnetic field. Mathematical Methods in the Applied Sciences, 41, 2350-2359.

Islam, A. W., Sharif, M. A. R. \& Carlson, E. S. (2012). Mixed convection in a lid driven square cavity with an isothermally heated square blockage inside. International Journal of Heat and Mass Transfer, 55, 5244-5255.

Kefayati, G. H. R., Gorji-Bandpy, M., Sajjadi, H. \& Ganji, D. D. (2012). Lattice Boltzmann simulation of MHD mixed convection in a lid-driven square cavity with linearly heated wall. Scientia Iranica, Transactions B: Mechanical Engineering, 19, 1053-1065.

Kishore, P. M., Rajesh, V. \& Verma, S. V. (2010). Effects of heat transfer and viscous dissipation on MHD free convection flow past an exponentially accelerated vertical plate with variable temperature. Journal of Naval Architecture and Marine Engineering, 7, 101-110.

Kumar, H. (2009). Radiative heat transfer with hydromagnetic flow and viscous dissipation over a stretching surface in the presence of variable heat flux. Thermal Science, 13, 163-169.

Müller, U. \& Bühler, L. (2001). Magnetofluiddynamics in Channels and Containers. Springer-Verlag Berlin Heidelberg, New York.

Nasrin, R. \& Parvin, S. (2011). Hydromagnetic effect on mixed convection in a lid-driven cavity with sinusoidal corrugated bottom surface. International Communication in Heat and Mass Transfer, 38, 781-789.

Nyabuto, R., Sigey, J. K., Okelo, J. A. \& Okwoyo, J. M. (2013). MagnetoHydrodynamics analysis of free convection flow between two horizontal parallel infinite plates subjected to constant heat flux. The SIJ Transactions on Computer Networks and Communication Engineering, 1, 79-83.

Rogers, D. F. (1992). Laminar Flow Analysis. CAMBRIDGE University Press.

Rudraiah, N. \& Barron, R. M. (1995). Effect of a magnetic field on free convection in a rectangular enclosure. International Journal of Engineering Science, 33, 1075-1084.

Yapıc1, K. \& Obut, S. (2015). Laminar mixed-convection heat transfer in a lid-driven cavity with modified heated wall. Heat Transfer Engineering, $36,303-314$. 


\section{Biographies}

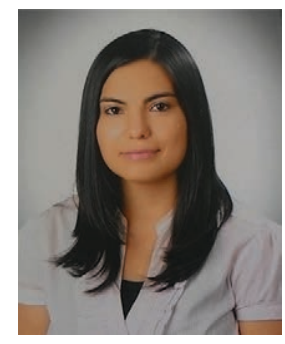

Merve Gürbüz received her $\mathrm{BSc}$ and $\mathrm{PhD}$ degrees in Mathematics from the Middle East Technical University, Turkey in 2012 and 2017, respectively. She is currently an Assistant Professor in the Department of Management, Başkent University, Turkey. Her research area is applied mathematics and numerical analysis.

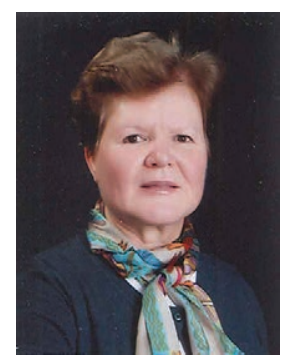

Münevver Tezer-Sezgin received her BSc in Mathematics from the Middle East Technical University, Turkey in 1974. She received her MSc and PhD degrees in Applied Mathematics in 1978 and 1980 from the University of Saskatchewan, Canada and the University of Calgary, Canada, respectively. She is retired and currently working as a part time Professor in the Department of Mathematics, Middle East Technical University, Turkey. She also has held one year visiting position at the University of Victoria, Canada, and short term visiting position at the Technical University of Darmstadt, Germany. She is the holder of Mustafa N. Parlar 1990 Research and 2014 Science Awards in applied mathematics and engineering. 
